\title{
PLANIFICACIÓN DE TRAYECTORIAS DE UN ROBOT MÓVIL MODULAR CON UN ÚNICO ACTUADOR
}

\author{
Adrián Peidró, Julio Gallego, Luis Payá, José María Marín, Óscar Reinoso \\ Laboratorio de Automatización, Robótica y Visión por Computador, Univ. Miguel Hernández, 03202 Elche \\ apeidro@umh.es, julio.gallego@alu.umh.es, lpaya@umh.es,jmarin@umh.es, o.reinoso@umh.es
}

\begin{abstract}
Resumen
Este artículo presenta un robot móvil modular que, con un único actuador, es capaz de controlar su posición y orientación a lo largo de un plano. Para ello, el cuerpo del robot consta de un conjunto de unidades de adhesión que puede fijar al entorno, de manera que su único motor hace pivotar al robot alrededor de la unidad de adhesión que se fije al entorno. Fijando de manera alterna unas u otras unidades de adhesión, el robot es capaz de moverse libremente por un plano, e incluso es capaz de realizar transiciones cóncavas entre planos perpendiculares o de combinarse con otros módulos idénticos para formar robots reconfigurables más complejos. En este artículo resolvemos el problema de planificación de trayectorias planas del robot propuesto, asumiendo que las trayectorias son poligonales e incluyen tramos estrechos dificiles de atravesar.
\end{abstract}

Palabras clave: robots con un único actuador, planificación de trayectorias, robots trepadores, robots modulares, pivote alternante, unidades de adhesión

\section{INTRODUCCIÓN}

Los Robots Móviles con un Único Actuador (RMUA) tienen la capacidad de explorar entornos con gran libertad de movimiento usando un único motor. Las ventajas de usar un único motor son menor coste, consumo energético, tamaño y peso. Gracias a estas ventajas, los RMUA tienen aplicaciones en tareas que requieran autonomía energética, miniaturización y navegación en áreas de difícil acceso, con fines de inspección, limpieza, rescate, o reconocimiento [12].

Como ilustra la Figura 1, la mayoría de RMUA encontrados en la literatura pueden clasificarse en tres tipos, en función de dos criterios: la dimensión de su espacio de trabajo, y la existencia de mecanismos binarios auxiliares que permiten al robot cambiar la dirección o tipología de su movimiento.

Los RMUA de tipo 1 se mueven por espacios de trabajo unidimensionales usando un único actuador, sin mecanismos auxiliares. En [11] se presenta un octópodo que puede avanzar hacia delante o atrás en línea recta usando un solo motor y un sistema de levas que coordina a sus ocho patas. De forma similar, en [3] y [1] se presentan sendos robots trepadores capaces de escalar verticalmente usando un único motor. En [13] se propone un robot capaz de avanzar en línea recta o escalar usando un movimiento ondulatorio generado por un único motor.

Los RMUA de tipo 2 pueden explorar espacios de trabajo con dimensión mayor a uno. Además de tener un actuador continuo principal, los RMUA de tipo 2 también disponen de mecanismos auxiliares binarios o todo-nada (embragues, imanes...) que les permiten modificar el efecto que su único actuador continuo ejerce sobre el movimiento global del robot, canalizando la energía motriz de una u otra forma para convertirla en avance en línea recta, salto, giro, etc. En esta línea, en [10] se presenta un robot móvil con dos ruedas de radio variable, actuadas por un único motor. Al activar cierto embrague, dicho motor también desplaza el centro de gravedad del robot, modificando el radio de las ruedas y generando un cambio en la dirección de avance. En [2] se propone un robot serpentino con un único actuador de tipo tendón. Aplicando selectivamente corrientes eléctricas a algunos de los segmentos que forman a este robot, es posible derretir temporalmente las uniones soldadas que los conectan, para modificar su geometría y lograr que el robot avance en línea recta o gire. En [6] se presenta un robot inspirado en cucarachas, con un único motor que le hace avanzar en línea recta o girar al modificar la rigidez de sus patas mediante aleaciones con memoria de forma.

Los RMUA de tipo 3 suelen ser robots subactuados con un único actuador y sin mecanismos auxiliares binarios, y pueden controlar su movimiento en espacios de trabajo con dimensión mayor a uno. Que estos robots realicen un movimiento $u$ otro (avance recto, giro, salto...) depende de las características de la señal de control aplicada a su único actuador (su amplitud, signo, frecuencia...). En [9] se presenta un robot capaz de nadar en línea recta o virar al excitar su único motor a frecuencia constante o variable, respectivamente. En [4] se propone un robot con un único actuador piezoeléctrico que le hace avanzar en línea recta o girar según se excite a una u otra de 


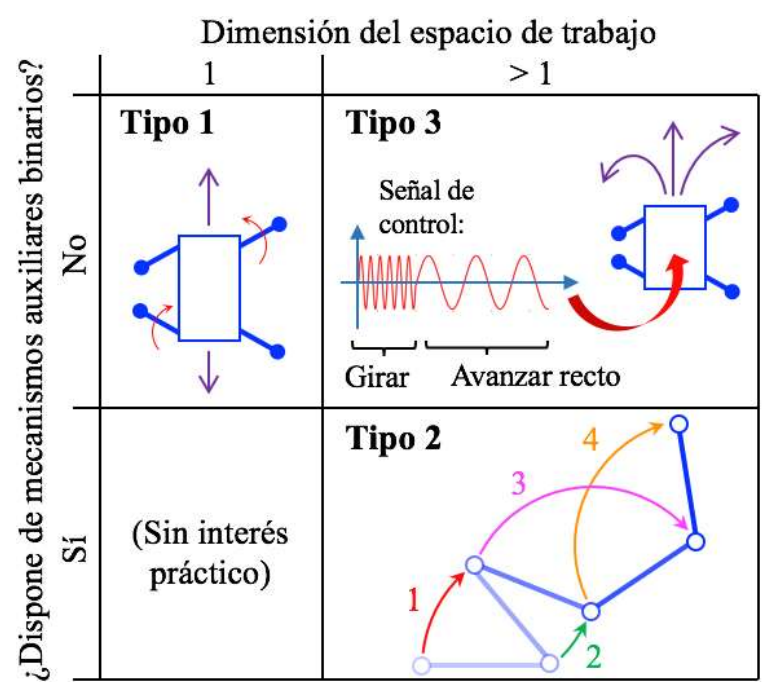

Figura 1: Clasificación de RMUA

sus frecuencias naturales. En [12] se presenta un hexápodo con patas de diferente rigidez, capaz de avanzar recto o girar en función de la forma del perfil de velocidades que se introduzca a su único motor. En [5] se propone una rueda robótica movida por un solo motor que le hace rodar en línea recta al oscilar entre dos posiciones límite, o bien le hace saltar al liberar la energía elástica de cierto muelle que se comprime al superar una de dichas posiciones límite. Finalmente, [14] presenta un robot saltador que puede realizar tres tipos de movimiento en función de la amplitud y el signo del giro de su único motor: saltar, levantarse tras el salto, y reorientarse para el siguiente salto.

Comparando los tres tipos de RMUA, se observa que los de tipo 1 son los más simples, pero únicamente pueden moverse en una dimensión. Por otro lado, los de tipo 3 pueden controlar su movimiento en espacios de trabajo de mayor dimensión, pero dicho control no es sencillo pues depende en gran medida de su dinámica subactuada [12]. En un término medio se encuentran los robots RMUA de tipo 2, que pueden controlar fácilmente su movimiento en espacios de trabajo de dimensión mayor a uno, a costa de incorporar mecanismos binarios auxiliares. Por este motivo, en este artículo se presenta el robot MASAR (véase la sección 2), que es un nuevo RMUA de tipo 2 capaz de moverse por planos usando un único motor $\mathrm{y}$ varias unidades de adhesión que pueden fijarse al entorno para modificar el punto alrededor del cual pivota el robot. En la sección 3 se resuelve el problema de planificación de trayectorias, para lograr que este robot siga trayectorias poligonales con estrecheces que coartan la amplitud de sus movimientos. La sección 4 ilustra el problema resuelto mediante experimentos, mientras que la sección 5 presenta las conclusiones y trabajos futuros.

\section{MASAR: UN ROBOT MODULAR CON UN ÚNICO ACTUADOR}

Esta sección presenta el robot MASAR (de su acrónimo en inglés: "Modular And Single-Actuator Robot"), que es un RMUA de tipo 2. Este robot, mostrado en la Figura 2, ha sido patentado por los autores ( $\mathrm{n}^{\mathrm{o}}$ ES2684377, fecha prioridad: 2017-03-31).
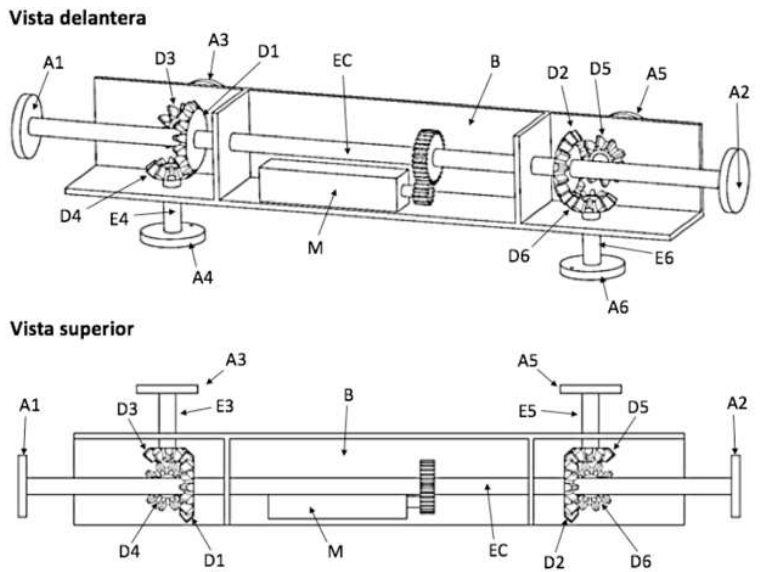

Figura 2: Robot MASAR, un RMUA de tipo 2

MASAR tiene un cuerpo B unido a un motor $\mathrm{M}$, que hace girar a un eje central EC. A cada lado del motor $M$ hay un juego de tres engranajes cónicos, de ejes concurrentes y perpendiculares (engranajes D1, D3, D4 a la izquierda de M, y D2, D5, D6 a su derecha). Cada engranaje cónico "Di" es solidario a una unidad de adhesión "Ai". Cada unidad de adhesión "Ai" puede estar pegada o despegada del plano con el que hace contacto. Cuando solo una unidad de adhesión está pegada al entorno, al accionar el motor $\mathrm{M}$ se produce la rotación de todo el robot alrededor de dicha unidad. Por ejemplo, al fijar A4 al entorno y accionar $\mathrm{M}$, el engranaje D1 rota sobre el engranaje D4, que está fijo (por estarlo A4), lo cual provoca la rotación de todo el robot alrededor de A4. Posteriormente, puede fijarse A6 y liberarse A4, con lo que el robot rotará alrededor de A6 de forma similar a como rotaba alrededor de A4. Repitiendo esta secuencia, en la que se fijan y liberan las unidades de adhesión de manera alterna, puede variarse el punto alrededor del que pivota el robot y producir así su avance por el plano, como ilustra la Figura 3.

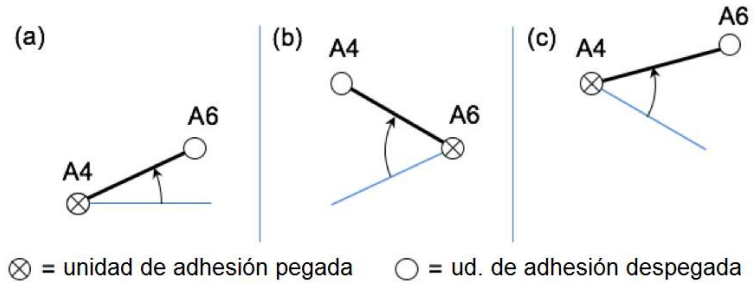

Figura 3: Locomoción del robot MASAR 
En [7] se presenta un robot similar al MASAR, que también emplea una secuencia de "pivote alternante" como la mostrada en la Figura 3 para avanzar por el plano utilizando un único actuador. No obstante, mientras que el robot presentado en [7] utiliza un mecanismo interno de correas, MASAR usa los juegos de engranajes cónicos mostrados en la Figura 2, y además ofrece más posibilidades de movimiento:

- Gracias a sus unidades de adhesión orientadas hacia planos perpendiculares, MASAR puede realizar transiciones cóncavas entre planos perpendiculares, lo cual le permite moverse entre el suelo, el techo, y paredes, como ilustra la Figura 4. Esto hace interesante al robot MASAR para explorar recintos de interior.

- Diversos módulos idénticos MASAR pueden colaborar y combinarse entre sí uniendo sus unidades de adhesión, formando robots modulares articulados que puedan desempeñar tareas complejas que un único módulo no podría. Por ejemplo, la Figura 5 ilustra cómo cuatro módulos MASAR podrían formar un brazo articulado de tipo serie para subir a una mesa. Un solo módulo no podría subir porque habría de realizar una transición convexa desde la pata de la mesa hacia su parte superior (y un módulo solo puede realizar transiciones cóncavas, como el paso desde el suelo a la pata de la mesa).

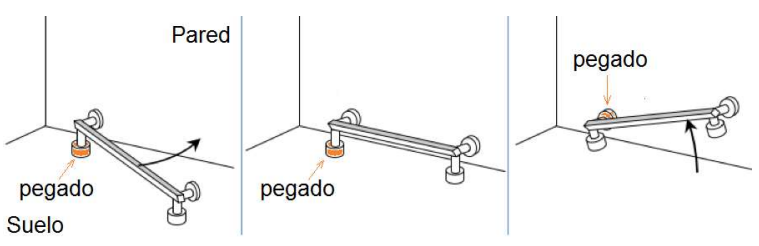

Figura 4: robot MASAR cambiando de plano

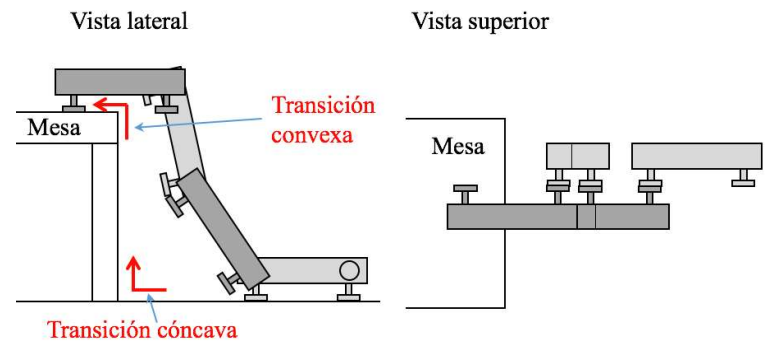

Figura 5: cuatro módulos MASAR colaborando para subir a una mesa

\section{PLANIFICACIÓN DE TRAYECTORIAS}

Esta sección aborda el problema de planificación de trayectorias planas del robot propuesto, asumiendo que la trayectoria a seguir es poligonal, estando formada por una secuencia de segmentos obtenidos de, por ejemplo, un algoritmo A*. Se considerarán los siguientes sub-problemas:

1. Cómo lograr que el robot siga una trayectoria poligonal sin obstáculos que restrinjan el movimiento del robot, es decir, no existe restricción en la amplitud de las rotaciones que puede realizar el robot.

2. Cómo lograr que el robot atraviese una sección estrecha, con estrecheces que restringen la amplitud de sus rotaciones.

3. Combinación de los dos problemas anteriores: seguir una trayectoria poligonal que pueda contener tramos con estrecheces.

Estos tres problemas serán resueltos geométricamente en las siguientes secciones.

\subsection{SEGUIMIENTO DE TRAYECTORIAS POLIGONALES}

La primera decisión a tomar es el tipo de locomoción que debe usar el robot para desplazarse a lo largo de trayectorias rectas sin restricciones u obstáculos que limiten la amplitud de sus movimientos. En ese caso, la locomoción más razonable es realizar giros de $180^{\circ}$, dado que éstos maximizan la distancia recorrida en un solo giro. Esto se ha ilustrado en la Figura 7.

Asumimos que, al inicio del trayecto poligonal, el robot comienza con una orientación cualquiera, y con su punto medio situado sobre el primer segmento del camino. Si este no fuera el caso, bastaría con rotar el robot alrededor de cualquiera de sus pivotes, hasta que su punto medio intersecara con el segmento.

Para comenzar el viaje a lo largo de la secuencia de segmentos mediante giros de $180^{\circ}$, el robot debe estar completamente alineado con el segmento inicial. Si ninguno de sus dos pivotes se encontrara inicialmente sobre la recta, serían necesarios dos giros para lograr la pose inicial deseada, como se explica seguidamente.

\subsubsection{Reorientación Inicial}

Como se ha mencionado anteriormente, inicialmente podría ocurrir que ambos pivotes no se encontraran sobre la recta a recorrer, el robot deberá entonces reorientarse para alinearse con la recta. Serán necesarios dos giros, como se ilustra en la Figura 6. 


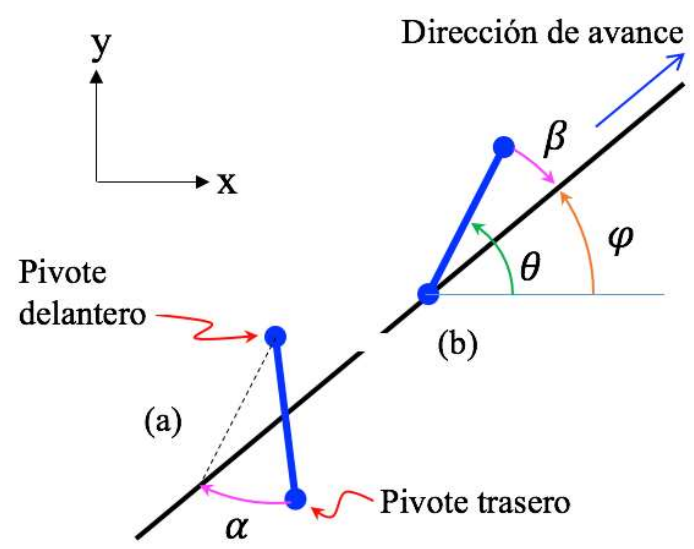

Figura 6: Reorientación inicial para alinear el robot con el primer segmento de la trayectoria poligonal

El primer giro, ilustrado en la Figura 6a, requiere buscar un punto sobre la recta cuya distancia al pivote delantero sea igual a la longitud del robot. Este punto se encuentra resolviendo la intersección entre la recta y una circunferencia centrada en el pivote delantero y con radio igual a la longitud del robot. Una vez encontrado tal punto de intersección, calculamos el valor de la rotación $\alpha$ que sitúa al pivote trasero sobre la recta. Considerando la recta como un vector apuntando hacia el punto al que debe moverse el robot, si el pivote de giro está situado a la derecha de este vector, el ángulo calculado será positivo (sentido antihorario). En caso contrario, será negativo (sentido horario), como en la Figura 6a.

La reorientación final, mostrada en la Figura 6b, parte de la situación previa, y será ejecutada tomando como pivote de giro el trasero, el cual ya se encuentra sobre el segmento. El ángulo a rotar el robot en este caso es $\beta=\theta-\varphi$, donde $\theta$ y $\varphi$ son, respectivamente, la orientación absoluta (es decir, con respecto al eje $\mathrm{x}$ ) del robot y de la recta. Ambos ángulos pueden calcularse mediante la función atan2.

\subsubsection{Movimiento Rectilíneo con Cambio de Dirección}

Cuando el robot está completamente sobre la línea, el movimiento a lo largo de ésta puede comenzar, realizándose mediante giros de $180^{\circ}$, alternando el pivote y el signo del ángulo de rotación:

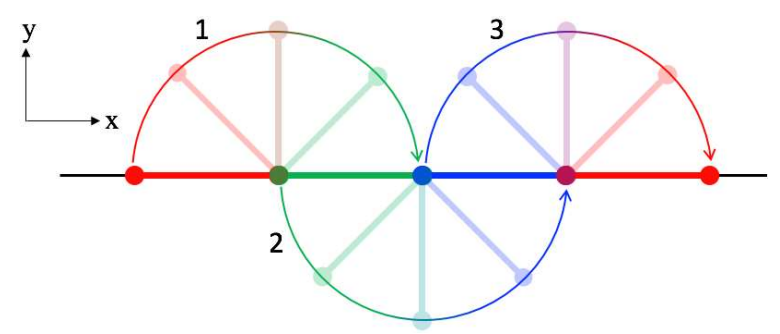

Figura 7: Locomoción mediante giros de $180^{\circ}$
Tras realizar un número determinado de rotaciones, la distancia entre el pivote delantero y el final de la recta estará por debajo de cierto umbral prefijado. En ese momento, el robot habrá de detener su avance e iniciará una secuencia de maniobras para cambiar al siguiente segmento de la trayectoria:

(a)

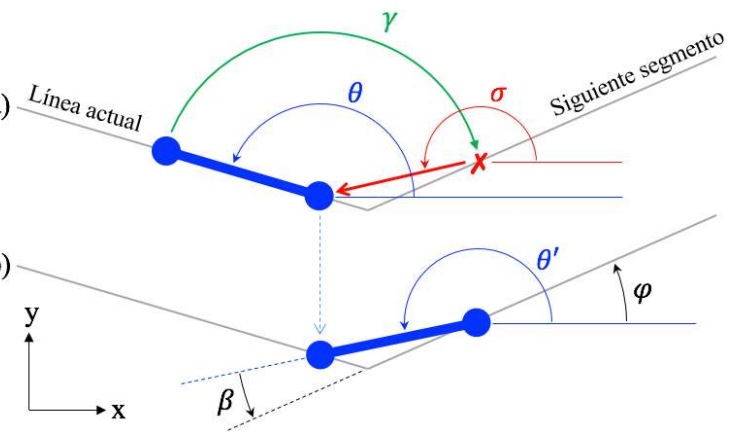

Figura 8: Cambio de dirección y reorientación

La primera maniobra consigue situar el pivote trasero en el siguiente segmento (Figura 8a). Esto se logra buscando un punto " $x$ " del siguiente segmento cuya distancia hasta el pivote delantero sea igual a la longitud del robot. De nuevo, esto consiste en resolver la intersección entre una recta y una circunferencia centrada en el pivote delantero y con radio igual a la longitud del robot, como en la Figura $6 a$.

Una vez encontrado el punto de intersección " $x$ ", se define un vector desde éste hasta el pivote delantero del robot: $\sigma$ denota el ángulo que dicho vector forma con el eje x. Además, también calculamos el ángulo $\theta$ que forma el robot con el eje horizontal. Con ello, el ángulo $\gamma$ que sitúa al pivote trasero sobre en punto de intersección será:

$$
\gamma=\sigma-\pi-\theta
$$

Finalmente, para situar el robot completamente sobre la nueva línea, habrá que rotar el robot un ángulo $\beta$ alrededor de su pivote delantero (véase la Figura $8 b$ ):

$$
\beta=\pi-\left(\theta^{\prime}-\varphi\right)
$$

siendo $\theta^{\prime}$ el nuevo ángulo entre el robot y el eje $\mathrm{x}, \mathrm{y}$ siendo $\varphi$ el ángulo entre la nueva línea y el eje $\mathrm{x}$. Tras completar el cambio de dirección, el robot continuará a lo largo de la nueva línea usando la locomoción descrita anteriormente: ejecutando giros de $180^{\circ}$ hasta alcanzar el final de esta nueva línea, después cambiará de nuevo la dirección de movimiento, etc.,... así hasta que el final de todo el camino poligonal sea alcanzado.

\subsection{ATRAVESANDO TRAMOS ESTRECHOS}

Esta sección se centra en la resolución de aquellas situaciones donde el robot ha de atravesar un tramo 
estrecho delimitado por dos paredes cercanas (véase la Figura 9). En tal caso, el movimiento definido en la sección 3.1 no es apropiado, ya que dentro de la estrechez no hay espacio suficiente para realizar las amplias rotaciones de $180^{\circ}$.

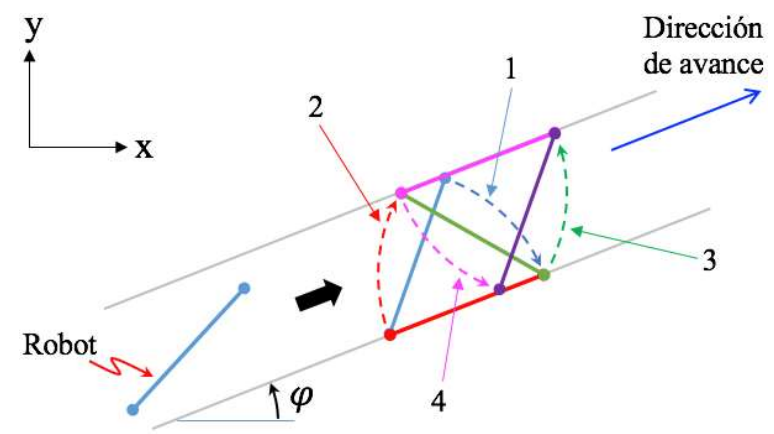

Figura 9: Atravesando un tramo estrecho

Para resolver la locomoción en estrecheces, asumiremos que éstas ocurren en un tramo horizontal. $\mathrm{Si}$ el robot encontrara una estrechez en un tramo oblicuo que forma un ángulo $\varphi$ con la horizontal (como en la figura anterior), entonces bastaría con rotar el problema un ángulo $-\varphi$, resolverlo según se explica en esta sección, y finalmente devolver la solución a su orientación inicial (rotando de nuevo todos los elementos un ángulo $\varphi$ ).

\subsubsection{Maniobra Inicial en la Estrechez}

La locomoción más rápida y eficiente para atravesar una estrechez consiste en colocar el cuerpo del robot completamente sobre una $\mathrm{u}$ otra pared de manera alterna, como ilustra la Figura 9. Este movimiento es la mejor opción ya que logra el máximo avance dentro de la estrechez, en cada paso. Para lograr este movimiento, el robot ha de iniciarlo con un pivote sobre cada pared.

El objetivo aquí es calcular el par de ángulos que debe rotar el robot (cada rotación alrededor de un pivote distinto) para alcanzar la posición inicial deseada.

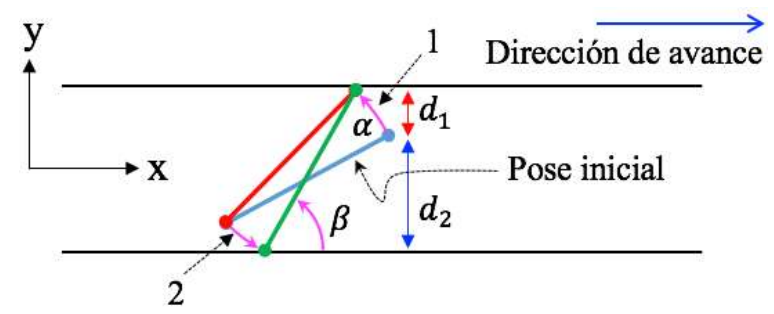

Figura 10: Situando cada pivote sobre una pared

Esta maniobra comienza buscando la mínima distancia $" \min \left\{d_{1}, d_{2}\right\} "$ desde cada pared hasta el pivote delantero del robot (véase la Figura 10):

$$
d_{i}=\frac{\left|a_{i} \cdot x_{\text {delantero }}+b_{i} \cdot y_{\text {delantero }}+c_{i}\right|}{\sqrt{a_{i}^{2}+b_{i}^{2}}}
$$

donde cada pared $i \in\{1,2\}$ viene definida por su ecuación implícita: $a_{i} x+b_{i} y+c_{i}=0$. La menor distancia obtenida indicará la pared sobre la que situar el pivote delantero.

Conocida la pared más cercana, hay que encontrar de nuevo un punto de ésta cuya distancia hasta el pivote trasero sea igual a la longitud del robot, como se realizó en las Figuras 6a y 8a. Este punto se obtiene resolviendo nuevamente la intersección entre la recta más cercana y una circunferencia centrada en el pivote trasero y de radio igual a la longitud del robot. Tras obtener este punto de intersección, se obtiene el ángulo $\alpha$ que se debe rotar el robot alrededor del pivote trasero para situar el delantero sobre el punto de intersección calculado.

Alcanzado este punto por el pivote delantero, debe realizarse de nuevo el mismo proceso para situar el pivote trasero sobre la pared opuesta, esta vez tomando como eje de rotación el pivote delantero.

\subsubsection{Movimiento a lo Largo de la Estrechez}

Tras alcanzar la posición inicial deseada, con cada pivote sobre una pared, el robot procede a desplazarse a lo largo de la estrechez hasta alcanzar su final. Este movimiento se consuma realizando siempre rotaciones con el mismo ángulo $\beta$, que es el ángulo formado entre el robot y las paredes cuando el robot tiene situado cada pivote en una pared diferente, como se indica en la Figura 10. Como se ha explicado antes, esta es la rotación óptima para avanzar por la estrechez, dado que produce el avance máximo a lo largo de la dirección de la estrechez.

El ángulo $\beta$ se calcula como en la Figura $6 \mathrm{~b}$, es decir, restando la orientación $\varphi$ de la recta de la orientación $\theta$ del robot. Después, el robot necesita alternar tanto el pivote de giro como el signo del ángulo $\beta$ en cada paso. Así avanzará situándose en la paredes de forma alternada, como se ilustra en la Figura 9.

\subsection{TRAYECTORIAS POLIGONALES CON ESTRECHECES}

Tras resolver los dos problemas previos, esta subsección analiza la posibilidad de combinarlos. Esta combinación está basada en una gestión "en línea" por parte del robot mientras se desplaza, es decir, a medida que avanza en su trayecto, si el robot encuentra una estrechez, un cambio de dirección, o rotaciones sin restricciones de $180^{\circ}$ a lo largo de una recta, ejecutará una de las acciones relevantes definidas en las subsecciones 3.1 y 3.2 anteriores. 
Llegado este punto, también es importante tener en cuenta cómo el robot es capaz de afrontar estrecheces, esto es, cómo entra y sale de ellas.

Tomando como punto de partida cualquier estrechez, para resolver esta situación se ha optado por definir una área estrecha virtual al comienzo y al final de la estrechez real. Estas áreas serán prolongaciones virtuales de la estrechez, como ilustra la Figura 11:

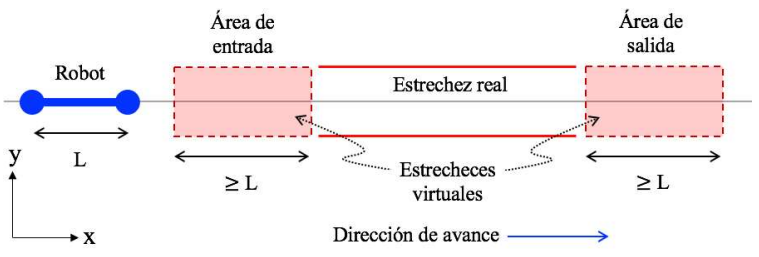

Figura 11: Entrando y saliendo de una estrechez

Por un lado, estudiando la entrada a la estrechez, cuando el pivote delantero del robot entre en la primera área virtual previamente mencionada (denotada como "Área de entrada” en la Figura 11), el robot entenderá que la estrechez está próxima. Entonces, se comportará como si ya estuviera dentro de ella: el robot ejecutará los movimientos correspondientes para situar sus pivotes en cada pared virtual (como en la Figura 10), y después avanzará usando la locomoción mostrada en la Figura 9, incluso si todavía se encuentra en la parte virtual de la estrechez. De este modo, está garantizado que el robot no intentará abordar la entrada de la estrechez ejecutando amplias rotaciones de $180^{\circ}$, las cuales pueden producir colisiones con las paredes de la estrechez antes de entrar en ella.

Por otro lado, para salir del tramo estrecho, se sigue un proceso similar. Esta vez el robot entiende que ha alcanzado el final de la estrechez cuando su pivote trasero abandona la segunda área virtual (denotada como "Área de salida”, en la Figura 11).

Es importante comentar que, a fin de evitar cualquier tipo de colisión, la longitud de ambas áreas virtuales debe ser mayor o igual que la longitud L del robot.

Finalmente, una vez el robot está completamente fuera de la estrechez, realiza la serie de movimientos mostrados en la Figura 6 para recuperar la completa alineación de su cuerpo con la recta actual que debe seguir recorriendo mediante rotaciones de $180^{\circ}$.

\section{EXPERIMENTOS}

Esta sección presenta un par de experimentos para ilustrar los problemas y soluciones analizadas en la sección anterior.

\subsection{COMPARACIÓN DE MODOS DE LOCOMOCIÓN}

En la sección 3 se eligió la locomoción óptima como aquélla en la que el robot avanza realizando giros de $180^{\circ}$, ya que éstos maximizan la distancia recorrida en un solo paso. A continuación, exploraremos la optimalidad de esta elección, analizando el tiempo total requerido por el robot para recorrer un segmento recto con una longitud 100 veces mayor que el robot. Como la Figura 12a ilustra, se asumirá que inicialmente el robot está perpendicular al segmento a recorrer. Al principio, el robot rota $\alpha / 2$ alrededor de uno de los pivotes, y a continuación comienza a rotar ángulos de " $-\alpha$ " o " $+\alpha$ " de manera alterna alrededor de uno $u$ otro pivote, hasta alcanzar el final de la trayectoria. El tiempo total $\tau$ para completar esta trayectoria vendrá dado por la siguiente expresión:

$\tau=T \cdot M(\alpha)+\frac{\alpha}{2 \omega}+\sum_{i=1}^{N(\alpha)} \frac{\alpha}{\omega}=T M(\alpha)+\frac{\alpha}{\omega}\left(N(\alpha)+\frac{1}{2}\right)$

donde se asume una velocidad angular constante $\omega$ (tomaremos $\omega=1 \mathrm{rad} / \mathrm{s}$ por simplicidad). $M(\alpha)$ es el número total de veces que las unidades de adhesión se pegan Y despegan durante la trayectoria, mientras que $N(\alpha)$ es el número de rotaciones de ángulo " $\alpha$ " Y $"-\alpha$ " necesarias para completar la trayectoria (tanto $M$ como $N$ dependen de $\alpha$ ). $T$ es el tiempo que una unidad de adhesión tarda en pegarse o despegarse.

La Figura 12b representa cómo varía $\tau$ con $\alpha$, para diferentes valores de $T$. Según se observa, para valores pequeños de $T$ (es decir, usando unidades de adhesión que tardan poco en pegarse o despegarse), el tiempo mínimo $\tau$ se logra para valores de $\alpha$ entre $50^{\circ}$ y $150^{\circ}$, aproximadamente (para cada $T$, el mínimo de $\tau$ se representa en la Figura $12 \mathrm{~b}$ como un punto negro). Para valores suficientemente grandes de $T$ (para $T>$ $7 \mathrm{~s}$ aproximadamente), es decir, para unidades de adhesión que toman algo de tiempo para pegarse o despegarse (por ejemplo, como ocurre con los imanes permanentes conmutables mecánicamente [8]), el mínimo tiempo $\tau$ se logra siempre aplicando rotaciones de $\alpha=180^{\circ}$ al robot. Cabe destacar en la Figura $12 \mathrm{~b}$ que, incluso para valores pequeños de $T$, el tiempo $\tau$ obtenido para $\alpha=180^{\circ}$ es solo ligeramente superior al tiempo mínimo que se obtendría al tomar el ángulo $\alpha$ óptimo para esos valores de $T$. Por tanto, $\alpha=180^{\circ}$ es siempre una excelente elección para la locomoción del robot, como sugiere la intuición.

\subsection{SEGUIMIENTO DE TRAYECTORIA DE EJEMPLO}

Esta sección muestra un ejemplo del problema descrito en la sección 3.3, en el que el robot debe seguir trayectorias poligonales que contienen algunos tramos estrechos, aplicando las soluciones expuestas 
en las secciones 3.1 y 3.2. Una trayectoria de ejemplo de este tipo se ilustra en la Figura 13, donde se indica la posición inicial y final del robot, así como diversas estrecheces. La Figura 13 muestra la secuencia de poses que el robot ha tomado al seguir esta trayectoria, secuencia obtenida aplicando los algoritmos y cálculos explicados en la sección 3. En la Figura 13, el robot siempre se representa como un segmento rojo.

\section{CONCLUSIONES Y TRABAJOS FUTUROS}

En este artículo, hemos descrito el robot MASAR: un nuevo robot modular con un único actuador. Este robot es un RMUA de tipo 2 porque dispone de elementos de adhesión binarios que pueden pegarse o despegarse del entorno, con el fin de producir la rotación del robot alrededor de diferentes ejes, logrando así controlar el movimiento del robot en dos o tres dimensiones usando un único actuador. En este artículo se ha resuelto el problema de seguimiento de trayectorias del robot MASAR, en el caso de trayectorias poligonales con posibles tramos estrechos que coartan la amplitud de los movimientos del robot. El modo de locomoción propuesto consiste en realizar rotaciones de $180^{\circ}$ cuando no existe riesgo de colisionar con obstáculos, con el fin de maximizar la distancia recorrida en un solo paso. En caso de encontrarse dentro de una estrechez, la locomoción óptima se logra rotando el robot hasta que sus dos pivotes tocan las paredes de la estrechez, ya que esta locomoción permite maximizar la distancia recorrida en un solo paso, sujeta a las restricciones de movimiento impuestas por los tramos estrechos.

En el futuro, abordaremos el problema de seguimiento de trayectorias del robot MASAR de manera más general, planteando un modelo cinemático del robot a nivel de velocidades, que nos permita ensayar diferentes leyes de control (por ejemplo, controladores PID) para seguir trayectorias planas arbitrarias, no necesariamente poligonales, en presencia de obstáculos del entorno. En el futuro también estudiaremos los problemas ilustrados en las Figuras 4 y 5 , es decir, la planificación de trayectorias que incluyan transiciones cóncavas entre planos perpendiculares, y la combinación y colaboración de varios módulos para formar robots articulados capaces de realizar tareas más complejas. Por último, actualmente también estamos desarrollando un prototipo funcional del robot MASAR.

\section{Agradecimientos}

Este trabajo ha sido respaldado por el Ministerio de Educación a través de una beca-colaboración concedida al segundo autor, y por el Ministerio de Economía a través del proyecto DPI 2016-78361-R.

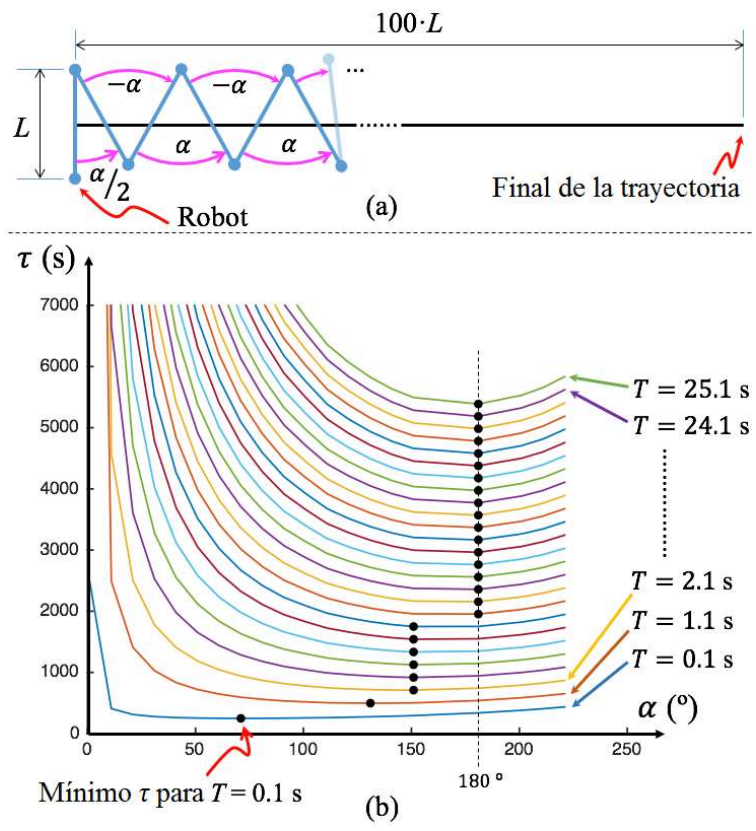

Figura 12: Tiempo $\tau$ para completar una trayectoria recta en función del ángulo de avance $\alpha$

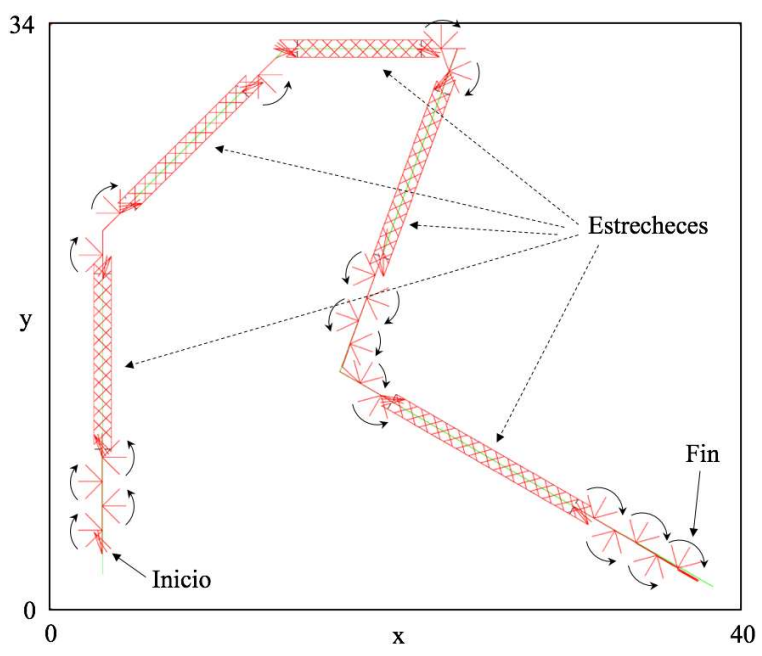

Figura 13: Ejemplo de seguimiento de trayectoria poligonal con estrecheces

\section{English summary}

\section{TRAJECTORY PLANNING OF A MODULAR MOBILE ROBOT WITH A SINGLE ACTUATOR}

\author{
Abstract \\ This paper presents a modular mobile robot that is \\ capable of controlling its position and orientation \\ along a plane using a single actuator. To that end, the \\ body of the robot has several adhesion units that can \\ be attached to the environment, such that the robot
}


will rotate about the fixed adhesion unit when powering its only actuator. By alternately attaching some or other adhesion units to the environment, the robot can freely move along a plane, and it is even capable of performing concave transitions between orthogonal planes, or combining with other identical modules in order to form complex reconfigurable robots. In this paper, we solve the trajectory planning of the proposed robot in a plane, assuming that the desired trajectories are polygonal and include narrow sections that are difficult to traverse.

Keywords: single-actuator robots, trajectory planning, climbing robots, modular robots, alternating pivot, adhesion unit

\section{Referencias}

[1] Birkmeyer, P., Gillies, A. G., Fearing, R. S., (2011). CLASH: Climbing vertical loose cloth. In 2011 IEEE/RSJ International Conference on Intelligent Robots and Systems (IROS), pp. 5087-5093.

[2] Cheng, N., Ishigami, G., Hawthorne, S., Chen, H., Hansen, M., Telleria, M., Playter, R., Iagnemma, K., (2010). Design and analysis of a soft mobile robot composed of multiple thermally activated joints driven by a single actuator. In 2010 IEEE International Conference on Robotics and Automation (ICRA), pp. 52075212.

[3] Choi, H. C., Jung, G. P., Cho, K. J., (2015). Design of a milli-scale, biomimetic platform for climbing on a rough surface. In 2015 IEEE International Conference on Robotics and Biomimetics (ROBIO), pp. 2205-2210.

[4] Dharmawan, A. G., Hariri, H. H., Foong, S., Soh, G. S., Wood, K. L., (2017). Steerable miniature legged robot driven by a single piezoelectric bending unimorph actuator. In 2017 IEEE International Conference on Robotics and Automation (ICRA), pp. 6008-6013.

[5] Ho, T., Lee, S., (2012). A novel design of a robot that can jump and roll with a single actuator. In 2012 IEEE/RSJ International Conference on Intelligent Robots and Systems (IROS), pp. 908913.

[6] Hoover, A. M., Burden, S., Fu, X. Y., Sastry, S. S., Fearing, R. S., (2010). Bio-inspired design and dynamic maneuverability of a minimally actuated six-legged robot. In 2010 3rd IEEE RAS and EMBS International Conference on Biomedical Robotics and Biomechatronics (BioRob), pp. 869-876.

[7] Mir-Nasiri, N., Siswoyo, H., Ali, M. H., (2018). Portable autonomous window cleaning robot. Procedia Computer Science, 133, 197-204.

[8] Peidró, A., Tavakoli, M., Marín, J. M., Reinoso, Ó., (2019). Design of compact switchable magnetic grippers for the HyReCRo structureclimbing robot. Mechatronics, 59, 199-212.

[9] Refael, G., Degani, A., (2015). Momentumdriven single-actuated swimming robot. In 2015 IEEE/RSJ International Conference on Intelligent Robots and Systems (IROS), pp. 2285-2290.

[10] Sfeir, J., Shammas, E., Asmar, D., (2014). Design and modeling of a novel single-actuator differentially driven robot. In 2014 IEEE/ASME International Conference on Advanced Intelligent Mechatronics (AIM), pp. 1079-1084.

[11] Soyguder, S., Alli, H., (2011). Motion mechanism concept and morphology of a single actuator tetrapod walking spider robot: the ROBOTURK SA-2 Robot. Industrial Robot: An International Journal, 38(4), 361-371.

[12] Zarrouk, D., Fearing, R. S., (2015). Controlled in-plane locomotion of a hexapod using a single actuator. IEEE Transactions on Robotics, 31(1), 157-167.

[13] Zarrouk, D., Mann, M., Degani, N., Yehuda, T., Jarbi, N., Hess, A., (2016). Single actuator wavelike robot (SAW): design, modeling, and experiments. Bioinspiration \& biomimetics, 11(4), 046004.

[14] Zhao, J., Xu, J., Gao, B., Xi, N., Cintron, F. J., Mutka, M. W., Xiao, L., (2013). MSU jumper: A single-motor-actuated miniature steerable jumping robot. IEEE Transactions on Robotics, 29(3), 602-614.

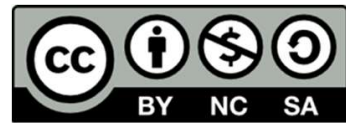
(C) 2019 by the authors. Submitted for possible open access publication under the terms and conditions of the Creative Commons Attribution CC BY-NC-SA 4.0 license (https://creativecommons.org/licenses/bync-sa/4.0/deed.es). 